\title{
THE CONTROL REVERSING ALGORITHM FOR AUTONOMOUS VEHICLES WITH PSD-CONTROLLED TRAILERS
}

\author{
Vladimír CVIKLOVIČ, Rastislav SRNÁNEK, Dušan HRUBÝ*, Marta HARNIČÁROVÁ \\ Slovak University of Agriculture in Nitra, Slovak Republic
}

\begin{abstract}
Driving a vehicle with a passive trailer has been the subject of numerous studies. Current control strategies are mostly used by differential geometry, linear algebra, fuzzy regulators, and artificial neural networks. The objective of this study is to design an algorithm for autonomous control of the tractor-trailer system reversing based on an algorithm using a PSD controller and to verify it on a simulated mathematical model. All parameters listed in the models and experiments are performed based on the existing tractor-trailer system. Dynamic models of steering and velocity control were identified and incorporated into the simulation. Result includes a stable operation of the steering without oscillating. The proposed algorithm can be implemented in microcontrollers without the need for high computing power.
\end{abstract}

Keywords: autonomous mobile robot; tractor-trailer system; hitch angle; controller

At present, under the agricultural sector conditions, the issue of soil compression with its negative effects is increasingly being discussed (Moinar and Shahgholi, 2019). The weight distribution of the system between vehicle and trailer reduces the negative soil compaction effect by distributing the resulting pressure between the three axles (Ansorge and Godwin, 2008). In terms of control, the main disadvantages of this solution include the longer length of tractor-trailer system and a higher difficulty of controlling the movement between obstacles and in narrow spaces. The issue of control is challenging because it is a 4-degree nonholonomic complex system, and the kinematics is complex to calculate (David and Manivannan, 2014). Since it is an unstable dynamic system, the input restrictions of which cause the vehicle to collide with the trailer, it is necessary to avoid exceeding the critical hitch angle. The hitch angle plays a critical role in jack-knife avoidance during slow backing up of vehicle-trailer systems (Chiu and Goswami, 2014).

Driving a vehicle with a passive trailer has been the subject of numerous studies. Current control strategies are mostly used by differential geometry, linear algebra, fuzzy regulators, and artificial neural networks (Hejase et al., 2018). In general, there is a tendency to use soft calculation techniques. Trailer systems have been studied in terms of nonlinear control theory (Bulgakov et al., 2017; Bulgakov et al., 2019). Initial research in the field of non-holonomic mobile robots has been extended to automotive-like robot applications. Much progress has been made in this area since the early 1990s. Several issues have been resolved, such as avoiding obstacles and parallel parking with a trailer (David and Manivannan, 2014). Furthermore, algorithms for automatic driving of vehicle to the charging station in the area of truck transport were also solved (Zobel, 2003).

Dynamics of a tractor-trailer system is asymmetric. The hitch angle is exponentially stable when the driving is directed forward. However, this case is not the subject of the study presented. When driving backwards, the system is unstable and requires a special control method (Pradalier and Usher, 2007). The static characteristics of certain dynamical systems can be linearized and thus the dynamic properties of members and circuits can be expressed using linear differential equations with constant coefficients. In such cases, these are systems with removable nonlinearity, therefore, it is assumed that the vicinity of the operating point on the nonlinear static characteristic of the controlled system can be replaced by a linear function. This assumption allows the use of theory of linear control, which is elaborated in detail and thus significantly simplifies the analysis and synthesis of control circuits (Balátě, 2003).

The objective of this study is to design an algorithm for autonomous control of the reversing of the tractor-trailer system based on an algorithm using a PSD (proportionalsummation-differential) controller and to verify it on a mathematical model in the simulation. Considering the proposed algorithm, there are expectations for low demands on computing power, no oscillations in the control and reliability for target points located in the second and third quadrants, where the trailer axle centre of coordinate system is located. It is assumed that it is possible to design an algorithm based on a linear PSD controller useable for autonomous vehicle control when reversing with a passive trailer, and that the angle between vehicle and trailer must be limited to avoid exceeding the hitch angle.

Contact address: Dušan Hrubý, Slovak University of Agriculture in Nitra, Faculty of Engineering, Institute of Electrical Engineering, Automation, Informatics and Physics, Tr. Andreja Hlinku 2, 94976 Nitra, Slovak Republic e-mail:dusan.hruby@uniag.sk 
The paper focuses on a consistent approach to the measurement of dynamics characteristics of the real tractor-trailer system as an input for simulation of the designed control algorithm when driving backwards. In this case, the use of linear regulator can provide satisfactory results.

\section{Material and methods}

\section{Kinematic model of tractor-trailer system}

In this case, a sprayer driven hydraulic motor and a spray tank are located on the trailer. The autonomous vehicle called SaMoRob (Fig. 1) includes an autonomous steering system with an energy unit with two separate hydraulic circuits. All parameters listed in the models and experiments will be observed based on the existing tractor-trailer system.

The kinematic model is shown in Fig. 2 with the basic system parameters. System states are described by $(x$, $y)$ the Cartesian coordinate of point $V$ in the global frame; $\theta_{V}$ and $\theta_{T}$ are the direction of tractor and trailer, respectively. Additionally, $\gamma$ is hitchangle defined as $\gamma=\theta_{T}-\theta_{V}$. The system is controlled via two inputs: the first is the linear velocity $v\left(\mathrm{~m} \cdot \mathrm{s}^{-1}\right)$ of point $V$, and the second is the steering angle $\varphi$ of the front wheels. This system can be described by the following equations (Pradalier and Usher, 2007):

$$
\begin{gathered}
\dot{x}=v \cos \left(\theta_{V}\right) \\
\dot{y}=v \sin \left(\theta_{V}\right) \\
\dot{\theta}_{v}=\omega_{v}=\frac{v_{V}}{L_{V}} \tan (\varphi) \\
\dot{\gamma}=-\left(\frac{v \tan (\varphi)}{L_{V}}\right)\left(\frac{h \cos (\gamma)}{L_{T}}+1\right)- \\
-\frac{v \sin (\gamma)}{L_{T}} \\
\dot{\theta}_{T}=-\frac{v\left[L_{V} \sin (\gamma)-h \tan (\varphi) \cos (\gamma)\right]}{L_{V} L_{T}}
\end{gathered}
$$

The first three equations $(1,2,3)$ of this system are the standard kinematics for a car-like vehicle. The equations 4 and 5 describe kinematics for a singleaxle trailer. In simulation, unit pixels represent their position.

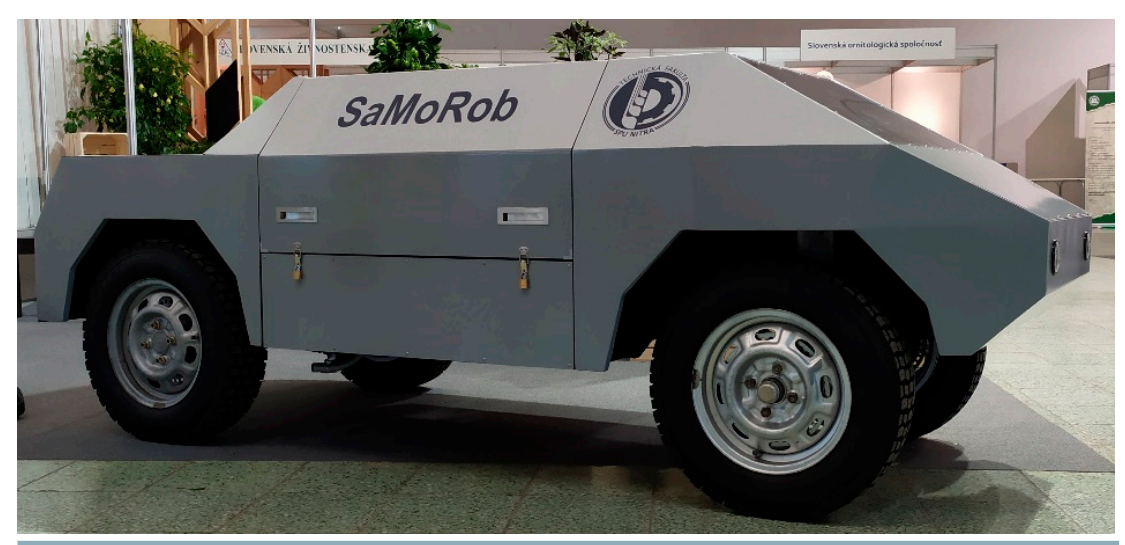

Fig. 1 Autonomous vehicle SaMoRob

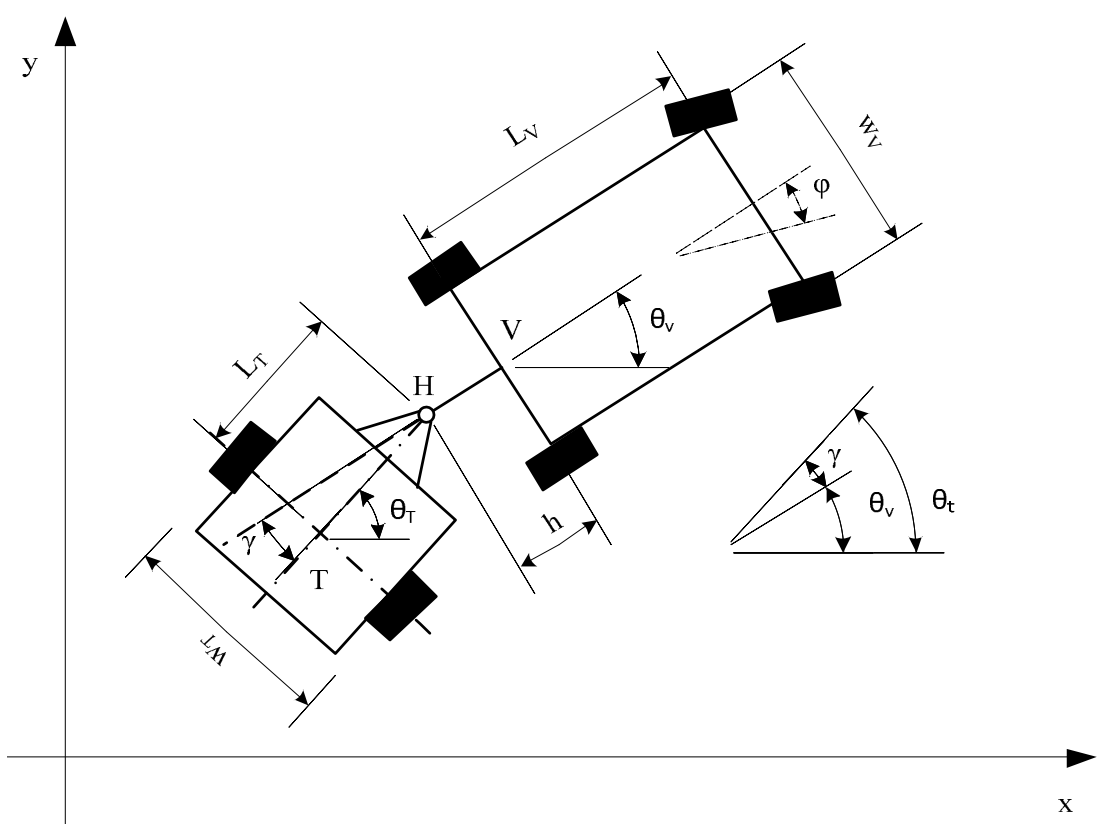

Fig. 2 Kinematic model of a tractor-trailer system

Physical parameters of single axle trailer system comprise longitudinal distance from vehicle rear axle to hitch $(h)-0.3 \mathrm{~m}$; vehicle wheelbase $\left(L_{v}\right)-1.7845$ $\mathrm{m}$; longitudinal distance from trailer axle to hitch $\left(L_{T}\right)-1.33 \mathrm{~m}$. Psychical vehicle parameter include: width $(w v)-0.975 \mathrm{~m}$; trailer width $\left(w_{T}\right)-1.09 \mathrm{~m}$. Further observed parameters include angles $\left(^{\circ}\right)$ : vehicle heading angle $\left(\theta_{V}\right)$; trailer heading angle $\left(\theta_{T}\right)$; steering angle $(\varphi)$; hitch angle $(\gamma)$.

It is necessary to know the work critical hitch angle for backing up the vehicletrailer system. That can be based on actual physical contact between vehicle and trailer kinematic criteria, in which the vehicle and trailer are perpendicular, or this can be an empirically determined threshold. A detailed elaboration of this issue was conducted by Chiu and Goswami (2014). In this case, critical hitch angle based on actual physical contact between vehicle and trailer is $79^{\circ}$. Empirically determined threshold of absolute critical hitch angle for tractor-trailer system is described mathematically (Chiu and Goswami, 2014):

$\gamma_{c, a}=-\arcsin \left[\frac{2 L_{T}}{\Delta x} \sin \left(\frac{\Delta x}{2 \operatorname{sgn}(\varphi) \sqrt{\left(L_{V} / \tan \left( \pm \varphi_{\max }\right)\right)^{2}+h^{2}}}\right]+\arctan \left(\frac{h \tan \left( \pm \varphi_{\max }\right)}{L_{V}}\right)\right.$ 
where:

$\gamma_{c r, a}-$ absolute critical hitch angle threshold beyond which no steering angle can reduce the hitch angle magnitude while moving backwards; $\Delta x$ - vehicle displacement

The main influencing factor is the range of steering angle $\varphi_{\max }$ because it is possible to change the movement speed smoothly and stably from zero the hydraulic system being controlled by the axial piston pump. The range of steering angle $\varphi_{\max }$ is from $-51^{\circ}$ to $+51^{\circ}$. The absolute critical hitch angle (Eq. 6) of tractor-trailer system in question is $25.46^{\circ}$.

\section{Identification of the system dynamics of an element}

The time course of steering and velocity of vehicle-trailer system is identified with a transient function according to the following mathematics. The concept of a transfer function is used to characterize the input-output relationship of components and subsystems that can be described by a linear, time-invariant, differential equation. The transfer function of the linear time-invariant system $(G(s))$ is defined as the ratio of the Laplace transform output $(Y(s))$ to the Laplace transform input $(U(s))$ with all initial conditions in the system set equal to zero (Fikar and Mikleš, 1999).

$$
G(s)=\frac{Y(s)}{U(s)}
$$

The iterative method of transfer estimation was employed. A normalized function $F(s)$ with output $y(t)$ and replacement function $\hat{F}(s)$ with output $x_{0}(t)$ can be described by complex functions:

$$
\begin{aligned}
& F(s)=\frac{1}{(1+T s)^{n}} \\
& \hat{F}(s)=\frac{1}{\left(1+T_{0} s\right)^{n}}
\end{aligned}
$$

Deviation $\in(t)$ between the actual and calculated output from the system is subtracted:

$$
\in(t)=x_{0}(t)-y(t)
$$

By introducing the steady time of transient characteristic as $T_{m}$ and replacing the error integral $P$ by geometric analogy, it is possible to get:

$$
P=\int_{T_{m}} \in(t) d t=A_{0} T_{m}
$$

where:

$A_{0}$ - height of fictive rectangular

By applying equality:

$$
\frac{1}{\left(1+T_{1} s\right)^{n}}=\frac{1}{\left(1+T_{0} s\right)^{n}}-A_{0}\left(1-e^{-T_{m^{s}}}\right)
$$

Correlation coefficient $R_{x y}$ is a criterion for an estimation of the system order:

$$
R_{x y}=\sqrt{\frac{1}{T_{m}} \int_{T_{m}} \in^{2}(t) d t}
$$

This integral for different orders of system was solved while selecting the best result of this integral, at which will be effective error lowest.

\section{System dynamics of steering}

Speed steering limit directly affects the control of the tractor-trailer system, and it is necessary to take it into account in terms of control model. In this case, steering is controlled via the stepper motor with the power gear. The controller is based on a special microcontroller circuit with feedback from the absolute optical encoder. Steering speed is not dependent on a vehicle velocity. The steering model was experimentally identified from the response of the unit jump response to the target steering angle.

The experiment was conducted on a rubber pad at zero vehicle speed, ensuring a high resistance between wheel and pad, which is rare in practice. The output was identified as a second-order system with two poles, two zeros and five free coefficients in the Laplace domain:

$$
G_{s}(s)=\frac{0.08915 s^{2}-12.19 s+925.2}{s^{2}+31.61 s+0.3681}
$$

Fit to estimation data is $99.41 \%$, indicating a very close dependence. This is also confirmed by the graph course in Fig. 3. Final prediction error is $0.008194 \%$ and mean square error is $0.008186 \%$. The identification used is based on Eqs 7-13.

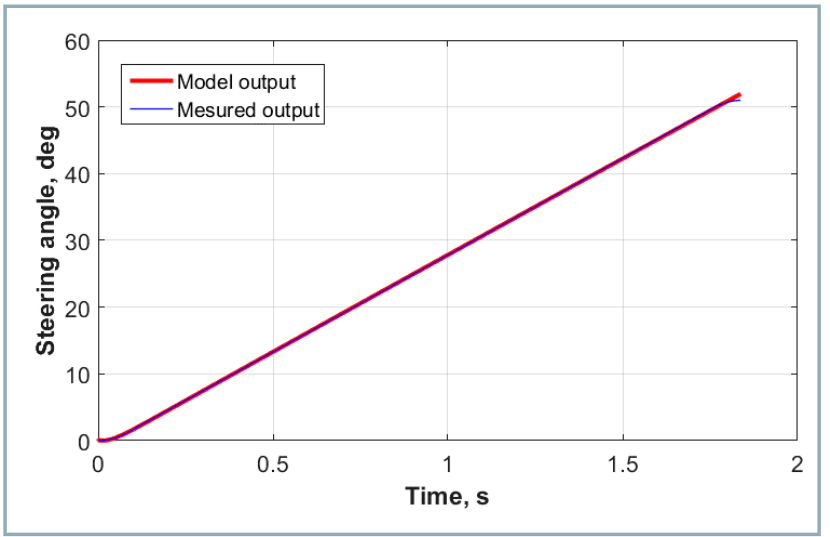

Fig. 3 Measured and simulated model output of steering angle

\section{System dynamics of velocity}

The vehicle speed control affects the automatic steering function in accordance with the steering velocity. The time course of the vehicle speed is determined as follows ( $\mathrm{Di}$ Martino, 2005):

$$
v_{v}=\frac{1}{m_{v}} \int\left[F_{t}-\left(F_{s}+F_{r}+F_{a}\right)\right] d t
$$

where:

$m_{v}$ - total vehicle mass ( $840 \mathrm{~kg}$ in this case); $F_{t}$ - traction force; $F_{s}$ - road slope force; $F_{r}$ - road load force; $F_{a}$ - aerodynamic drag force 
If we consider a slope inclination of $10^{\circ}$, a speed of $1 \mathrm{~m} \cdot \mathrm{s}^{-1}$, then the acceleration is $1.545 \mathrm{~m} \cdot \mathrm{s}^{-2}$ with the current vehicle concept. The transient response was measured and identified due to the need for accurate model as described below.

The vehicle speed is regulated electronically by a stepper motor with a gearbox controlled by a microcontroller in the same way as in case of steering. The vehicle acceleration is changed by the microcontroller firmware. Based on the factors that can be determined, the mechanical parameters and performance of vehicle do not affect the speed control characteristics.

The same results were confirmed by an experiment performed on a rubber pad glued to a flat concrete surface in laboratory. This was to minimize the effect of tire slippage during the measurement. The steering angle $\varphi$ were set to zero. The measurement of dynamic properties took place when the speed jumped from zero to $1.4 \mathrm{~m} \cdot \mathrm{s}^{-1}$. The measurements confirmed the same course of the current speed when moving forwards and backwards. The current vehicle position was measured using four quadrature encoders. Each encoder measured the angular velocity of one wheel. The travel and speed information were processed by a microcontroller according to the standard odometry mathematics. The sampling period was $50 \mathrm{~m} \cdot \mathrm{s}^{-1}$, one wheel revolution corresponds to 34 pulses. The tires used were $165 / 70$ R13, a dynamic radius of $98 \%$ of the wheel radius was considered.

The velocity loop was identified as the second order system with two poles, two zeros and five free coefficients in the Laplace domain as described below (own design):

$$
G_{V}(s)=\frac{0.009626 s^{2}-1.264 s+91.25}{s^{2}+33.18 s+0.2505}
$$

Fit to estimation data is $99.55 \%$, suggesting a very close dependence. This was also confirmed by the course in Fig. 4. Final prediction error is $0.000917902 \%$ and mean square error is $0.0001188 \%$.

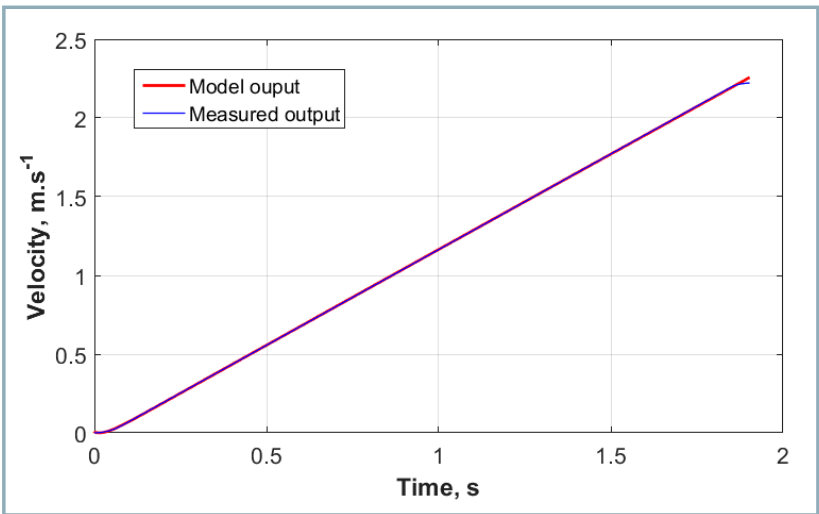

Fig. 4 Measured and simulated model output of the vehicle velocity

\section{System dynamics of digital regulation}

In this case, a PSD controller in incremental form was used. The algorithm does not determine the whole value $u(k T)$ of the action variable at a given moment, but its change $\nabla u(k T)=u(k T)-u[(k-1) T]$, i.e., the increment $\nabla u(k T)$ compared to the important $u[(k-1) T]$ in the previous step. The incremental algorithm of PSD controller in the form of a differential equation is as follows (Balátě, 2003):

$$
\begin{gathered}
\nabla u(k T)=u(k T)-u[(k-1) T] \\
=k_{R}\left\{\{e(k T)-e[(k-1) T]\}+\frac{T}{T_{I}}\left\{\sum_{i=1}^{k} e(i T)-\sum_{i=1}^{k-1} e(i T)-\right\}+\right. \\
\left.+\frac{T_{D}}{T}\{e(k T)-2 e[(k-1) T]+e[(k-2) T]\}\right\}= \\
=k_{R}\left\{\{e(k T)-e[(k-1) T]\}+\frac{T}{T_{I}}\{e(k T)\}+\right. \\
\left.+\frac{T_{D}}{T}\{e(k T)-2 e[(k-1) T]+e[(k-2) T]\}\right\}
\end{gathered}
$$

where:

$$
\begin{aligned}
u(k T)=k_{R}\{e(k T) & \left.+\frac{T}{T_{1}} \sum_{i=1}^{k} e(i T)+T_{D} \frac{e(k T)-e[(k-1) T]}{T}\right\} \\
u[(k-1) T] & =k_{R}\left\{e[(k-1) T]+\frac{T}{T_{1}} \sum_{i=1}^{k-1} e(i T)+\right. \\
& \left.+T_{D} \frac{e[(k-1) T]-e[(k-2) T]}{T}\right\}
\end{aligned}
$$

By introducing the parameters $q_{0}, q_{1}, q_{2}$ :

$$
\nabla u(k T)=q_{0} e(k T)+q_{1} e[(k-1) T]+q_{2}[(k-2) T]
$$

where:

$$
\begin{gathered}
q_{0}=k_{R}\left(1+\frac{T}{T_{1}}+\frac{T_{D}}{T}\right) \\
q_{1}=-k_{R}\left(1+2 \frac{T_{D}}{T}\right) \\
q_{2}=k_{R} \frac{T_{D}}{T}
\end{gathered}
$$

Adjustable parameters are $k_{R}, T_{1}$ and $T_{D}$. Their values can be substituted by the values obtained according to the selected criteria by any optimization procedure for a continuous PID (proportional-integral-derivative) controller. The discrete PSD controller generates action interventions $\nabla u(k T)$ based on the measured current value of the control deviation $e(k T)$ and its memorized past values $e[(k-1) T], e[(k-2) T]$. The inverse rectangular method was used in the derivation for the integral substitution.

The control process quality is characterized by a transition process, i.e., the way in which the controlled variable $y(t)$ or the control deviation $e(t)$ passes from one steady-state to another steady-state. This process can be assessed either by a controlled variable, or a control deviation. Indirect indicators of regulatory quality include integral quality criteria, such as the integration of the control deviation at time $t(s)$ (Balátě, 2003):

$$
Q_{1}=\int_{0}^{\infty}[y(t)-y(\infty)] d t
$$


The criterion result is a number indicating the area under the transient component of the control deviation. Minimization of the Q1 indicator can be a criterion for the optimal setting of controllers. Said indicator Q1 is advantageous (very simple) for aperiodic transition processes. However, the disadvantage of this indicator is that, during the periodic transition process, the areas lying below the axis are subtracted, so that even systems with large and long-lasting overshoots show very good values of this indicator. For this shortcoming, the transient component quadratic values of the control deviation were taken into account, which formally converted all areas to positive (Balátě, 2003):

$$
Q_{2}=\int_{0}^{\infty}[y(t)-y(\infty)]^{2} d t
$$

A higher-quality system (a system with small deviations and a shorter regulation time) has a lower value of the Q2 criterion.

\section{Results and discussion}

The designed control algorithm is based on hitch angle regulation. Firstly, it is necessary to calculate the distance $d$ and angle $\delta$ between point $T$ and final point C. Subsequently, the desired hitch angle $\gamma_{z}$ is calculated as follows (own design):

$$
\gamma_{z}=\frac{\delta}{d} \times n
$$

where:

$n$ - desired hitch angle gain dependent on the kinematic model of the tractor-trailer system. With larger $n$, the path circle radius will be also larger. The value of desired hitch angle $\gamma_{z}$ is input of the PSD controller and the desired steering angle $\varphi$ is its output

The algorithm solves a critical element, which is the hitch angle stabilization; therefore, it is not necessary to additionally solve the gamma angle regulation by a special controller. The hitch angle is limited to a calculated critical value of $25.46^{\circ}$. The flow chart of proposed algorithm is shown in Fig. 5. It is based on a PSD controller and mathematically described as Eq. 27, which is based on Eqs 17-23.

$$
\begin{gathered}
u_{n}=u_{n-1}+K\left(e_{n}-e_{n-1}\right)+T_{1} \times T_{0} \times e_{n}+\frac{T_{D}}{T_{o}} \\
\left(e_{n}-2 \times e_{n-1}+e_{n-2}\right)
\end{gathered}
$$

where:

$u_{n}$ - action variable; $u_{n-1}$ - previous action variable; $e_{n}$ control deviation; $e_{n-1}, e_{n-2}-$ control deviations of previous states

The block diagram of designed simulation model is shown in Fig. 6. Application of dynamic models to the algorithm testing is crucial in terms of the correct debugging

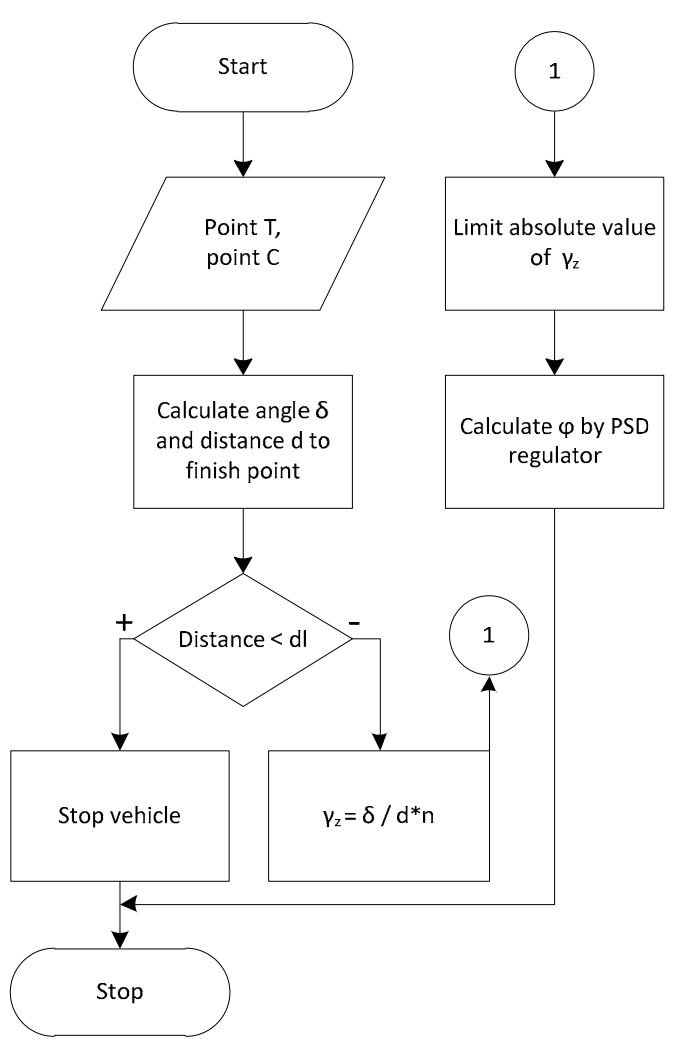

Fig. 5 Control algorithm for reversing the tractor-trailer system (own design)

of developed control algorithm, because the time delay of the set parameters directly affects the model results. The most significant is the effect on driving speed. The control algorithm inputs are actual point $T$, desired point $C$ and actual hitch angle $\gamma$. Its outputs are required steering angle $\varphi_{Z}$ and required vehicle velocity $v_{Z}$ (velocity of point $\eta$. Real values at time were calculated by transfer function for steering and velocity control $G_{V}(s)$ and $G_{V}(s)$ (Eqs 15 and 16). Finally, positions of the tractor-trailer system and hitch angle were calculated using Eqs 1-5.

The optimal coefficients of PSD controller found based on the algorithm are provided in Fig 7. The coefficient $K$ was changed from 0 to 2 in steps of 0.1 . The coefficients $T_{l}, T_{D}$ and $T_{O}$ were changed in the range from 0 to 1 in steps of 0.01 . The controller update period is $6 \mathrm{~ms}$. The vehicle speed when reversing is $0.5 \mathrm{~m} \cdot \mathrm{s}^{-1}$. The error was calculated according to Q2 criterion (Eq. 25). The criterion according to Eq. 24 is not applicable due to system predispositions to oscillations.

The integral Q2 control quality criterion for fine-tuning the PSD controller coefficients was used for reversing to six different target points (Table 1). The target points were selected randomly with an even distribution in space behind the tractor-trailer system. The initial position of the trailer axle centre $T$ was $[0,0]$, hitch angle $\gamma=0$, steering angle $\varphi=0$ and velocity of the point $P$ was zero. If the absolute value of distance from the target position is less than $360 \mathrm{~mm}$, the algorithm ends and the vehicle is stopped. 


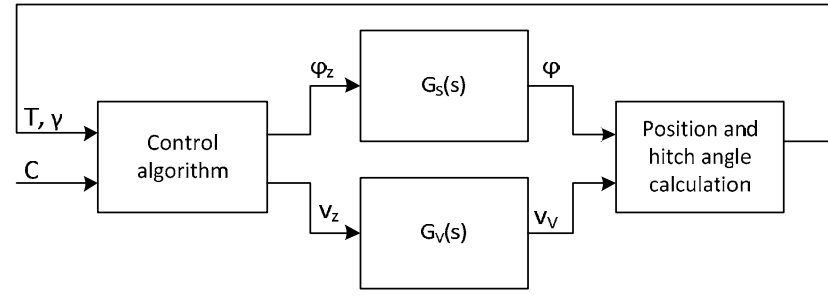

Fig. 6 Designed simulation model (own design)

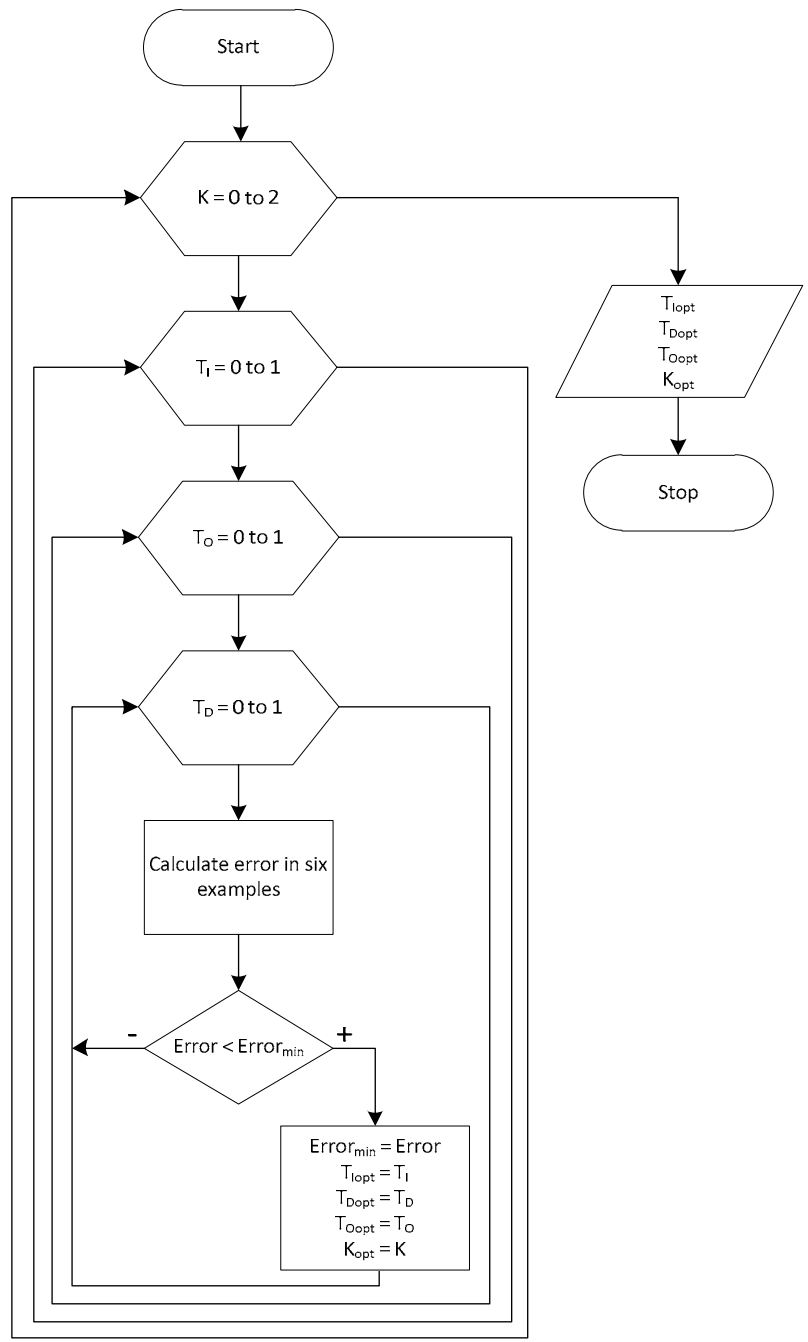

Fig. 7 Designed algorithm for finding of the optimal coefficients (own design)

The lowest value of the criterion was reached for parameters $T_{1}=0.06, T_{D}=0.15, T_{O}=0.05$ and $K=1.5$. Figure 8 depicts the waveforms indicating a stable operation of the steering without oscillating the steering wheel, which is advantageous in case of backlash in the steering. The hitch angle did not reach a critical value in any case. The oscillation occurred a few seconds before reaching the target position because of a rapid change in the set value of the hitch angle due to the increase in the angle between the truck and the target.
Table 1 Six final points for the algorithm testing and finding the optimal coefficients

\begin{tabular}{|l||c|c|}
\hline Path number & Location $x(\mathrm{~mm})$ & Location y $(\mathrm{mm})$ \\
\hline \hline $\mathbf{1}$ & -10128 & 600 \\
\hline $\mathbf{2}$ & -5808 & -6990 \\
\hline $\mathbf{3}$ & -9858 & 5910 \\
\hline $\mathbf{4}$ & -9828 & -4110 \\
\hline $\mathbf{5}$ & -3378 & 8070 \\
\hline $\mathbf{6}$ & -2598 & -7140 \\
\hline
\end{tabular}

Figure 9 shows the six paths of tractor-trailer system. The target position was achieved smoothly in all cases. Based on the aforementioned, it can be concluded that reversing of tractor-trailer systems can be controlled by a linear regulator. However, obstacles were not taken into account in this study.

A better illustration of reversing control in simulations is provided in Fig. 10; four different examples of the tracks with tractor-trailer skeletons were plotted.

In comparison to the current state reflected in actual reference literature, the advantage of proposed algorithm is the absence of a special controller for the hitch angle, simplifying the entire control system. As shown in Figs 8-10, linear operation without jitter was achieved. It is assumed that this fact will be positively reflected in real testing.
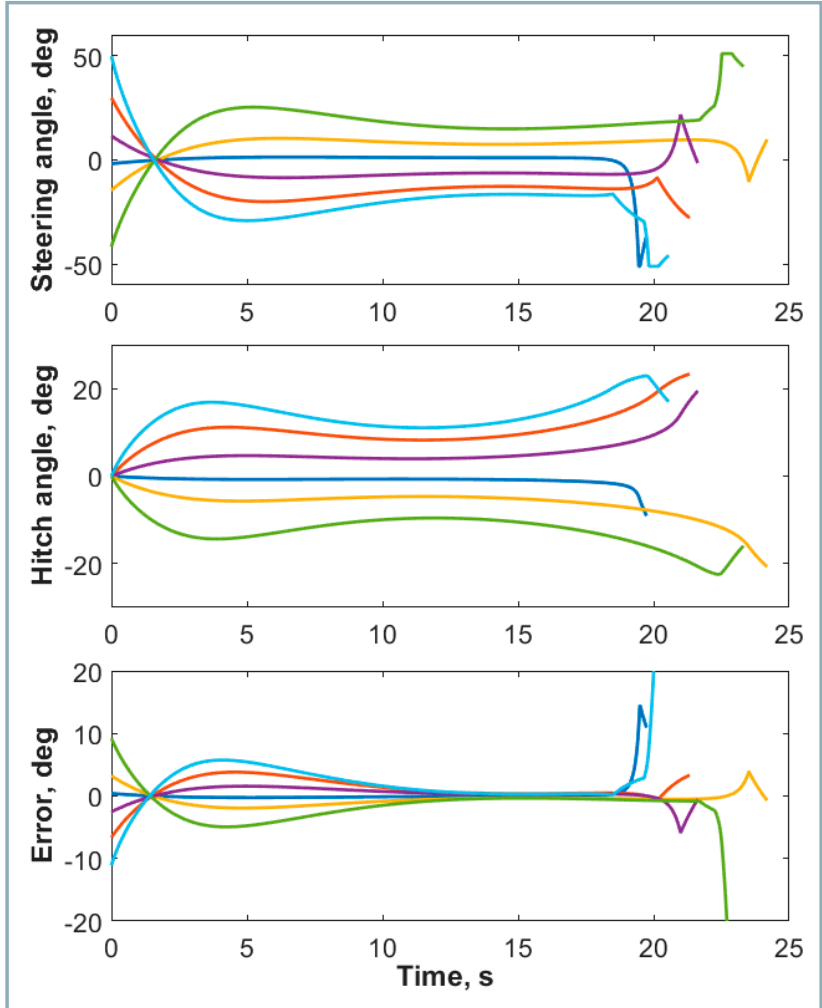

Fig. 8 Waveforms of steering angle, hitch angle and hitch angle error for six paths

blue - path 1 , red - path 2 , orange - path 3 , violet - path 4 , green - path 5, light blue - path 6 (own design) 


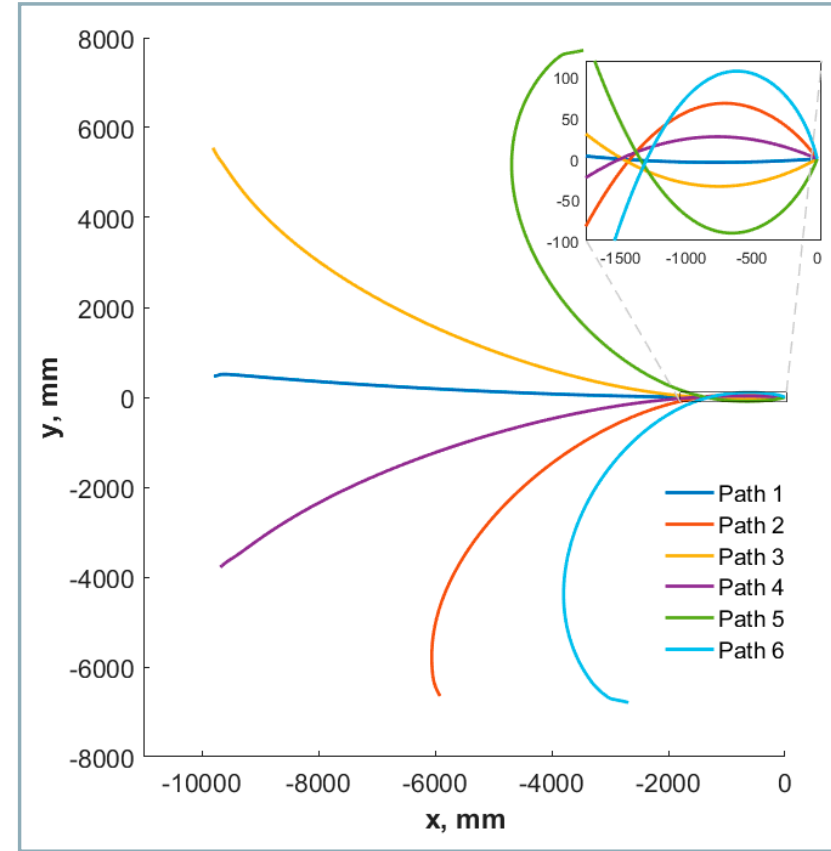

Fig. 9 Paths of the trailer axle centre leading to the destination point (own design)

\section{Conclusions}

Research regarding the steering of tractor-trailer systems is constantly being updated. Various solutions are employed; however, these require a robust control system in a majority of cases. The paper presented provides a workable control algorithm, which can be implemented in microcontrollers without the need for high computing power.

It has been shown that it is possible to use a control algorithm based on a PSD controller for reversing the vehicle with a passive trailer without an additional solution to prevent a jack-knife. This demonstrated the assumption that non-holonomic systems can be controlled via conventional linear regulators. However, it is necessary to find a controlled variable in the system that can be described by linear transient characteristics. In this case, the regulation is focused directly on the hitch angle.

In the described algorithm, the target point can be changed during the control according to the requirements resulting from the constraints due to the spatial distribution of obstacles. However, this topic deserves a separate contribution and thus will be published in detail in the future.

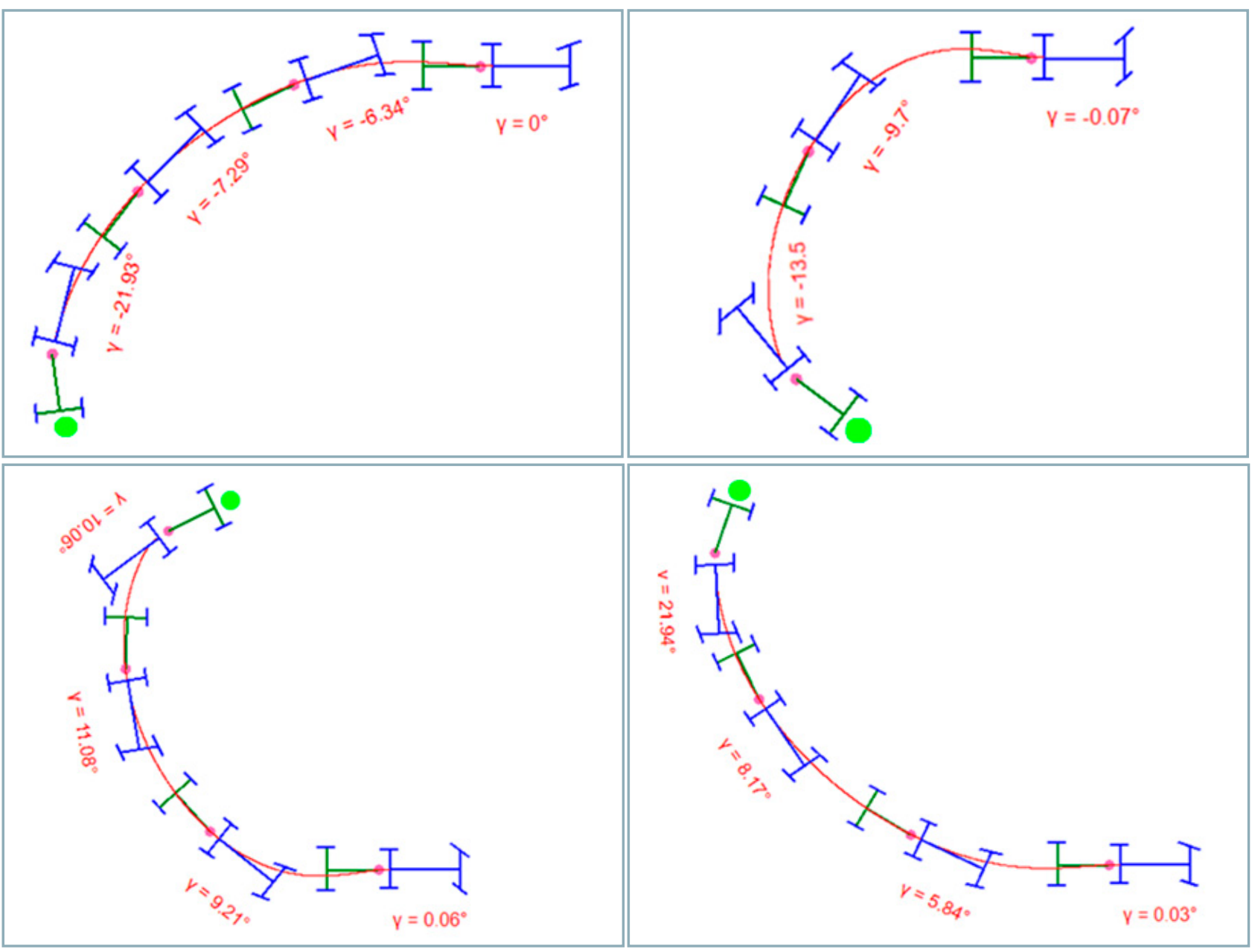

Fig. 10 Paths of the tractor-trailer system with location details (the target point is green) (own design) 


\section{Acknowledgements}

This paper and the research explored in it would not have been possible to conduct without the exceptional support from the entire Department of Electrical Engineering, Automation and Informatics of the Faculty of Engineering of the Slovak University of Agriculture in Nitra. There was a large number of people helping us with their enthusiasm, knowledge and attention to detail, providing us conditions for work despite the pandemic situation.

\section{References}

ANSORGE, D. - GODWIN, R. 2008. The effect of tyres and a rubber track at high axle loads on soil compaction - Part 2: Multi-axle machine studies. In Biosystems Engineering, vol. 99, no. 3, pp. 338-347.

BALÁTĚ, J. 2003. Automatic control. Prague : BEN - Technická literature, 664 pp. ISBN 9788073001483. (In Czech: Automatické rízení).

BULGAKOV, V. - IVANOVS, S. - ADAMCHUK, V. - ANTOSHCHENKOV, R. 2019. Investigations of the dynamics of a four-element machineand-tractor aggregate. In Acta Technologica Agriculturae, vol. 22 no. 4, pp. 146-151.

BULGAKOV, V. - ADAMCHUK, V. - NADYKTO, V. - KYURCHEV, V. - NOZDROVICKÝ, L. 2017. Theoretical consideration of the controllability indicator of machine-tractor unit movement. In Acta Technologica Agriculturae, vol. 20, no. 1, pp. 11-18.

CHIU, J. - GOSWAMI, A. 2014. The critical hitch angle for jackknife avoidance during slow backing up of vehicle-trailer systems. In Vehicle System Dynamics, vol. 52, no. 7, pp. 992-1015.
DAVID, J. - MANIVANNAN, P. V. 2014. Control of truck-trailer mobile robots: a survey. In Intelligent Service Robotics, vol. 7, no. 4, pp. 245-258.

DI MARTINO, R. 2005. January. Modelling and simulation of the dynamic behaviour of the automobile. Available at: https://tel.archives-ouvertes.fr/tel-00736040/document

FIKAR, M. - MIKLEŠ, J. 1999. System identification. Bratislava : STU Press, 112 pp. ISBN 8022711772. (In Slovak: Identifikácia systémov). HEJASE, M. - JING, J. - MAROLI, J. M. - BIN SALAMAH, Y. FIORENTINI, L. - ÖZGÜNER, Ü. 2018. Constrained backward path tracking control using a plug-in jackknife prevention system for autonomous tractor-trailers. In $201821^{\text {st }}$ International Conference on Intelligent Transportation Systems (ITSC), pp. 2012-2017.

MOINAR, A. - SHAHGHOLI, G. 2019. The effect of tractor driving system type on its slip and rolling resistance and its modelling using ANFIS. In Acta Technologica Agriculturae, vol. 22, no. 4, pp. 115-121.

PRADALIER, C. - USHER, K. 2007. A simple and efficient control scheme to reverse a tractor-trailer system on a trajectory. In Proceedings 2007 IEEE International Conference on Robotics and Automation, pp. 2208-2214.

ZOBEL, D. 2003. Trajectory segmentation for the autonomous control of backward motion for truck and trailer. In IEEE Transactions on Intelligent Transportation Systems, vol. 4, no. 2, pp. 59-66. 